\title{
GÉNERO, SIGNIFICADOS E CONSUMO DE PORNOGRAFIA EM PORTUGAL: UM ESTUDO MISTO
}

\author{
Rita Castro ${ }^{1}$ e Samuel Lins ${ }^{1}$ \\ 1Universidade do Porto, Portugal.a.r.f.c@hotmail.com; samuellins@fpce.up.pt
}

\begin{abstract}
Resumo. Introdução: O consumo de pornografia é uma realidade e tem vindo a crescer. Objetivo: O objetivo deste estudo foi compreender se os significados da pornografia são influenciados pelo género e analisar se o género está relacionado com o consumo e atitudes face à pornografia. Método: Este estudo adotou um método misto. Para conhecer os significados da pornografia, utilizou-se a técnica de associação livre de palavras, e para mensurar as atitudes face à pornografia, utilizou-se a Escala de Atitudes Face ao Uso de Materiais Pornográficos. Os dados qualitativos foram tratados com o programa informático IRaMuTeQ, e os quantitativos com o Statistical Package for the Social Sciences. Resultados: Os resultados revelaram que os significados da pornografia se agrupam em três classes distintas: 1) Indústria pornográfica e aspetos negativos; 2) Categorias pornográficas; e 3) Propósitos do uso e aspetos positivos, e que as classes estavam associadas a diferentes géneros. Os resultados também mostraram que há diferenças no consumo e nas atitudes face a pornografia em função do género. Conclusão: Assim, tanto a análise qualitativa como a análise quantitativa coincidiram, revelando resultados semelhantes.
\end{abstract}

Palavras-chave: Pornografia; Género; Métodos Mistos; Portugal; Sexualidade.

\section{GENDER, MEANINGS AND CONSUMPTION OF PORNOGRAPHY IN PORTUGAL: A MIXED STUDY}

\begin{abstract}
Introduction: The consumption of pornography is a reality and has been growing. Objective: The aim of this study was to understand whether the meanings of pornography are influenced by gender and to analyze whether gender is related to consumption and attitudes towards pornography. Method: This study adopted a mixed method. In order to know the meanings of pornography, the technique of free word association was used, and to measure attitudes towards pornography, the Attitudes Scale towards the Use of Pornographic Materials was used. Qualitative data were treated with the computer program IRaMuTeQ, and quantitative data with the Statistical Package for the Social Sciences. Results: The results revealed that the meanings of pornography are grouped into three distinct classes: 1) Porn industry and negative aspects; 2) Pornographic categories; and 3) Purposes of use and positive aspects, and that the classes were associated with different genders. The results also showed that there are differences in consumption and attitudes towards pornography based on gender. Conclusion: Thus, both the qualitative analysis and the quantitative analysis coincided, revealing similar results.
\end{abstract}

Keywords: Pornography; Gender; Mixed-Methods; Portugal; Sexuality.

\section{INTRODUÇÃO}

O consumo de pornografia é muito comum na nossa sociedade (Blais-Lecours, VaillancourtMorel, Sabourin, \& Godbout, 2016). A tecnologia, a Internet, alavancou o acesso aos conteúdos pornográficos, com a facilidade de acesso, disponibilidade, e anonimato conferido (Cooper, 1998). De facto, hoje é mais fácil do que nunca aceder a conteúdos pornográficos (Cooper, Morahan-Martin, Mathy, \& Maheu, 2002). 
Contudo, a investigação sobre pornografia na população em geral escasseia (Alarcón, Iglesia, Casado, \& Montejo, 2019; Morgan, 2011). Em Portugal, as investigações são poucas. Uma pesquisa realizada em fevereiro de 2020 com os termos "pornography" e "Portugal", nas bases de dados EBSCOhost, Scopus e SciELO, encontrou apenas sete publicações focalizadas no estudo da pornografia.

Ademais, a definição de pornografia ainda é inconsistente (Short, Black, Smith, Wetterneck, \& Wells, 2012). Esta difere entre culturas (Watson \& Smith, 2012) e entre indivíduos da mesma cultura (Brown, Durtschi, Carroll, \& Willoughby, 2017; Hald, Seaman, \& Linz, 2014). Portanto, nesta investigação, adotou-se a seguinte definição: pornografia é qualquer material que veicule imagens sexualmente explícitas, como exposição da genitália e/ou representações de comportamentos sexuais, com intento de provocar excitação sexual ou fantasia (Morgan, 2011; Rasmussen, 2016; Short et al., 2012).

A consciência do impacto das considerações sociais e culturais, como estigmas e estereótipos presentes na sociedade relativamente à sexualidade (Alves, Amâncio, \& Alferes, 2008; Blais-Lecours et al., 2016; Tarrant, 2016) impele à consideração do género na investigação sobre pornografia.

Assim, esta investigação, através de um método misto, objetivou compreender se os significados da pornografia são influenciados pelo género e analisar se o género está relacionado com o consumo e atitudes face à pornografia.

\subsection{Consumo de pornografia}

O consumo de pornografia tem crescido. Em 2019, o maior site pornográfico do mundo, o Pornhub, registou 42 mil milhões de visitas (Pornhub, 2019). E, este crescimento tenderá a manter-se, devido à evolução da tecnologia, com o cinema pornográfico 5D e a realidade virtual (Medium, 2017).

Em Portugal, o consumo de pornografia é uma realidade. As últimas estatísticas do Pornhub mostram que, em 2018, Portugal ocupou a $39^{a}$ posição no ranking mundial, sendo $29 \%$ dos visitantes mulheres (Público, 2019). 


\subsection{Género}

O género é uma variável de relevo no estudo da sexualidade. Por exemplo, estudos mostram que o género masculino tem atitudes mais permissivas face ao sexo do que o género feminino (Alferes, 1997; Gomes, Fernandes, Ribeiro, \& Cardoso, 2018; Lam \& Chan, 2007; Lo \& Wei, 2005; Luquis, Brelsford, \& Rojas-Guyler, 2012). E, no consumo de pornografia, as diferenças de género têm emergido de forma regular (Hald et al., 2014). Os homens revelam mais consumir materiais pornográficos, sobretudo na Internet (BlaisLecours et al., 2016; Brown et al., 2017; Cooper et al., 2002; Gomes, Fernandes, Ribeiro, \& Cardoso, 2018; Gomes, Fernandes, Ribeiro, Cardoso, \& Ramos, 2018; Lo \& Wei, 2005; Morgan, 2011; Raposo, 2018; Træen et al., 2006). Contudo, alguns estudos indicam inexistência de diferenças entre os géneros (Carvalho, Czop, Rocha, Nobre, \& Soares, 2018; Carvalho et al., 2013; Guerra, Gouveia, Sousa, Lima, \& Freires, 2012).

Portanto, os resultados não são claros quanto ao papel do género no consumo de pornografia. Por um lado, o facto do consumo aparecer, maioritariamente, associado ao homem pode dever-se às diferentes preferências de estímulos sexuais entre homens e mulheres. Os homens excitam-se mais com estímulos eróticos visuais, encontrados nos sites pornográficos, revelando corpos e sexo explicitamente, e as mulheres excitam-se mais com estímulos textuais, escritos, que traduzem interações relacionais (Cooper et al., 2002; Esteves, 2018). Por outro lado, as mulheres podem não assumir o consumo de pornografia tanto quanto os homens, devido aos estereótipos e estigmas que ainda existem na sociedade (Alves et al., 2008; Blais-Lecours et al., 2016; Tarrant, 2016). Por exemplo, através do duplo padrão sexual, ou seja, de normas sociais que determinam comportamentos sexuais distintos em função do género (Reiss, 1964), havendo um julgamento diferenciado para o mesmo comportamento sexual, com favorecimento da permissividade e liberdade sexual do género masculino (Alves et al., 2008; Zaikman \& Marks, 2017).

\section{MÉTODO}

A presente investigação recorreu ao método misto, usando metodologias de investigação qualitativa e quantitativa. A utilização do método misto permite mais robustez na análise, através da confirmação e complementaridade que oferece para a compreensão dos fenómenos. Através da integração de metodologias qualitativas e quantitativas, é possível maior segurança nas conclusões derivadas, do que apenas com uma metodologia unilateral 
(quantitativa ou qualitativa) (Small, 2011). A metodologia mista também tem sido utilizada em outros estudos na área da sexualidade (Lorvick et al., 2012; Pachankis, Buttenwieser, Bernstein, \& Bayles, 2013).

\subsection{Participantes}

Recolheu-se uma amostra por conveniência de 665 adultos portugueses (406 do género feminino, 253 do género masculino e seis de outro género), com uma média de idade de 25.72 anos $[D P=8.85$, mínimo $=18(n=93)$, máximo $=75(n=1)]$. A maioria dos participantes reportou orientação heterossexual ( $n=540: 81.20 \%$ ), e encontrar-se num relacionamento amoroso ( $n=393$ : 59.10\%). Ademais, tratou-se de uma amostra, maioritariamente, estudante ( $n=375: 56.39 \%)$, com habilitações ao nível do ensino superior ( $n=418: 62.86 \%)$, e rendimentos mensais até $1000 €(n=509: 76.54 \%)$.

\subsection{Materiais}

Os dados foram recolhidos através de um questionário de autorrelato composto por quatro secções, a seguir detalhadas. Todos os participantes responderam ao questionário na íntegra.

\subsubsection{Significados da Pornografia}

Utilizou-se a técnica de associação livre de palavras. Esta técnica projetiva permite recolher de forma livre e simples os construtos latentes associados a um objeto (Abric, 2003). Pediuse aos participantes que enumerassem as cinco primeiras palavras/expressões que thes surgiam na mente ao pensar em pornografia. E, pediu-se que notassem a positividade de cada expressão/palavra numa escala tipo Likert de cinco pontos $(1=$ muito negativa; $5=$ muito positiva).

\subsubsection{Posicionamento Face ao Consumo de Pornografia}

Solicitou-se aos participantes que selecionassem uma opção de entre as seguintes: Eu sou contra o consumo de pornografia; Eu sou a favor do consumo de pornografia; e Eu sou indiferente. 


\subsubsection{Consumo de Pornografia}

Colocaram-se duas questões: 1) Alguma vez consumiu materiais pornográficos (e.g., revistas, livros, sites pornográficos)? (sim; não); e 2) Quão frequentemente consome materiais pornográficos? ( 1 = nunca; 10 = mais de uma vez por dia).

\subsubsection{Atitudes Face à Pornografia}

Usou-se a Escala de Atitudes Face ao Uso de Materiais Pornográficos (Guerra, Andrade, \& Dias, 2004), com 27 itens, que mede as atitudes face à pornografia através de duas escalas. A escala de atitudes de diferencial semântico, com três itens, num contínuo de cinco pontos, com as palavras Nocivo-Benéfico; Desagradável-Agradável; e Imprudente-Prudente $(\alpha=$ .87). E, a escala de efeitos positivos, com 12 itens (e.g., É certo aumentar o prazer recorrendo a pornografia; $\alpha=.92$ ), e efeitos negativos, com 12 itens (e.g., A utilização de pornografia é prejudicial, pois vicia; $\alpha=.87$ ), respondida numa escala tipo Likert de cinco pontos ( 1 = discordo totalmente; $5=$ concordo totalmente).

\subsubsection{Questionário Sociodemográfico}

Recolheram-se informações como idade, género, orientação sexual, se estava num relacionamento, situação profissional, habilitações académicas e rendimento mensal.

\subsection{Procedimento}

Nesta investigação, a metodologia qualitativa, com a técnica de associação livre de palavras, revelava-se importante, por permitir conhecer mais aberta e espontaneamente as dimensões associadas à pornografia. A metodologia quantitativa complementou a abordagem qualitativa com a análise da relação entre os significados da pornografia e o género, consumo e atitudes face à pornografia.

\subsection{1 Ética e Deontologia}

Apresentou-se aos participantes o consentimento informado. Pedia-se que respondessem a um questionário sobre o consumo de pornografia e ressalvava-se a garantia de confidencialidade e anonimato, a participação voluntária e a possibilidade de desistência sem qualquer penalização a qualquer momento. Deixou-se ao dispor um e-mail para eventuais contactos. 


\subsubsection{Recolha de Dados}

O processo de recolha de dados decorreu online, entre setembro de 2018 e fevereiro de 2019, através da plataforma SurveyMonkey. O questionário foi divulgado via e-mail institucional para várias universidades e institutos superiores do país, bem como, organizações relacionadas com a área da sexualidade humana e divulgado nas redes sociais (Facebook e LinkedIn).

\subsubsection{Análise Qualitativa}

A análise qualitativa iniciou-se com a realização da redução de palavras por três juízes independentes. A redução de palavras (i.e., eliminar artigos/proposições, transformar verbos na forma infinitiva, adotar para palavras similares a mais frequente) é um procedimento habitual na técnica de associação livre de palavras (Rosenberg \& Jones, 1972).

Finda a etapa da redução de palavras, recorreu-se ao software de análise textual IRaMuTeQ (Ratinaud, 2009). Este software apresenta elevada precisão estatística e oferece uma ampla variedade de análises lexicais. Nomeadamente, a análise da frequência das palavras e, posterior, organização através da Classificação Hierárquica Descendente (Camargo \& Justo, 2013).

Para a análise da frequência das palavras, criou-se a nuvem de palavras. Esta ilustra através de uma imagem a frequência com que as palavras ocorrem, sendo maiores as palavras mais evocadas.

Para compreender a organização dos significados da pornografia, realizou-se a Classificação Hierárquica Descendente, que organiza os segmentos textuais semelhantes em classes. E, desta organização resulta um dendrograma indicando a quantidade e a composição lexical das classes através do agrupamento das palavras, tendo cada palavra um valor de Chi-quadrado $\left(x^{2}\right)$, que quanto maior mais significativa é a associação da palavra com a classe que integra (Camargo \& Justo, 2013).

Ainda se analisou se as classes estariam associadas com o género (feminino, masculino ou outro); com o posicionamento face ao consumo de pornografia (a favor, contra ou indiferente); e com a frequência de consumo de pornografia. Para analisar a relação da frequência de consumo de pornografia com os significados da pornografia transformou-se a variável frequência de consumo, inicialmente ordinal, numa variável categorial. Tomando a 
mediana como ponto de corte obtiveram-se duas categorias: (1) Consumo menos frequente, integrando as opções desde $1=$ nunca até $6=$ duas/três vezes por mês ( $n=335: 57.70 \%$ ); e (2) Consumo mais frequente, integrando as opções desde $7=$ uma/duas vezes por semana até $10=$ mais de uma vez por dia $(n=246: 42.30 \%)$.

\subsubsection{Análise Quantitativa}

A análise quantitativa realizou-se no Statistical Package for the Social Sciences (SPSS) (versão 25). Realizou-se análise descritiva, correlação R de Pearson e teste-t de Student, para analisar o consumo de pornografia e a relação do género com o consumo e atitudes face à pornografia.

\section{RESULTADOS}

\subsection{Significados}

A nuvem de palavras mostrou que as dez palavras mais frequentes foram: sexo $(n=522)$, prazer $(n=210)$, masturbação $(n=130)$, mulheres $(n=87)$, nudez $(n=79)$, excitação $(n=$ $62)$, vídeos $(n=62)$, orgasmo $(n=62)$, homem $(n=49)$ e fantasia $(n=45)$ (Figura 1$)$. Contudo, a análise em função do género denotou algumas diferenças.

No género feminino as cinco palavras mais citadas foram: sexo $(n=334)$, prazer $(n=109)$, masturbação ( $n=73)$, nudez $(n=56)$ e homem $(n=41)$. E, no género masculino foram: sexo $(n=186)$, prazer $(n=98)$, masturbação $(n=56)$, mulheres $(n=49)$ e mamas $(n=28)$.

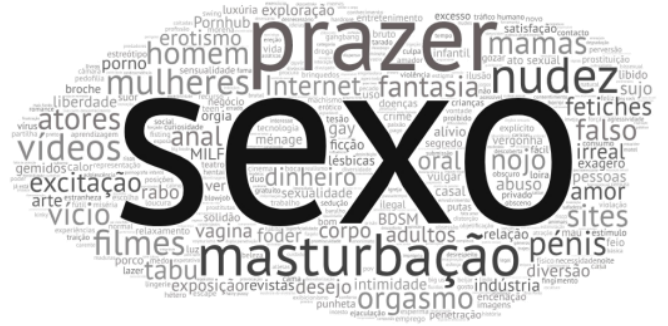

Figura 1. Nuvem de palavras dos significados da pornografia.

De modo a complementar a análise de frequência, realizou-se a análise textual, que considerou as 3325 palavras recolhidas, perfazendo 421 formas diferentes.

A Classificação Hierárquica Descendente integrou $72.48 \%$ do material recolhido, ou seja, 482 Segmentos de Texto (ST), tendo emergido duas ramificações. A primeira integra as classes 1 e 2 , e a segunda a classe 3 (Figura 2). 


\begin{tabular}{|c|c|c|c|c|c|}
\hline \multicolumn{2}{|c|}{$\begin{array}{c}\text { Classe } 1 \\
\text { Indústria pornográfica e } \\
\text { aspetos negativos } \\
(45.64 \%) \\
220 \mathrm{ST}\end{array}$} & \multicolumn{2}{|c|}{$\begin{array}{c}\text { Classe } 2 \\
\text { Categorias pornográficas } \\
(17.84 \%) \\
86 \mathrm{ST}\end{array}$} & \multicolumn{2}{|c|}{$\begin{array}{c}\text { Classe } 3 \\
\text { Propósitos do uso e aspetos } \\
\text { positivos } \\
(36.51 \%) \\
176 \mathrm{ST}\end{array}$} \\
\hline Palavras & $x^{2}$ & Palavras & $x^{2}$ & Palavras & $x^{2}$ \\
\hline Dinheiro & 34.65 & Anal & 111.21 & Prazer & 81.88 \\
\hline Internet & 30.63 & Vagina & 106.15 & Excitação & 78.27 \\
\hline Falso & 24.35 & Pénis & 104.38 & Orgasmo & 40.02 \\
\hline Nojo & 20.49 & Mamas & 103.37 & Masturbação & 39.74 \\
\hline Indústria & 19.22 & Oral & 55.35 & Diversão & 23.23 \\
\hline Atores & 18.69 & Rabo & 51.83 & Mulheres & 20.25 \\
\hline Filmes & 18.44 & MILF & 42.23 & Amor & 17.75 \\
\hline Vergonha & 18.44 & Broche & 42.23 & Relaxamento & 14.14 \\
\hline Videos & 17.68 & Ménage & 31.81 & Proibido & 12.35 \\
\hline Exposição & 16.55 & Gangbang & 26.93 & Sexo & 11.83 \\
\hline Sites & 12.16 & Foder & 24.07 & Privado & 9.12 \\
\hline Negócio & 12.16 & Blowjob & 18.57 & Tesāo & 8.78 \\
\hline Doenças & 12.16 & Pornhub & 18.13 & Fantasia & 8.63 \\
\hline Abuso & 10.92 & Fisting & 13.90 & & \\
\hline Imagem & 9.69 & dupla penetraçăo & 13.90 & & \\
\hline Exploraçāo & 8.46 & Amador & 13.90 & & \\
\hline Estereótipo & 8.46 & Esperma & 13.32 & & \\
\hline \multirow[t]{2}{*}{ Revistas } & 8.10 & Bruto & 13.32 & & \\
\hline & & Loira & 8.99 & & \\
\hline "Género feminino & 28.89 & *Género masculino & 16.88 & \multirow{4}{*}{$\begin{array}{l}{ }^{*} \text { Posic. a favor } \\
{ }^{*} \text { Consumo menos } \\
\text { freq. }\end{array}$} & 20.16 \\
\hline *Posic. Indiferente & 25.74 & ${ }^{*}$ Género outro & 8.99 & & 12.88 \\
\hline *Posic. Contra & 14.81 & ${ }^{\star}$ Posic. a favor & 10.17 & & \\
\hline $\begin{array}{l}{ }^{*} \text { Consumo menos } \\
\text { freq. }\end{array}$ & 14.75 & ${ }^{\star}$ Consumo mais freq. & 17.28 & & \\
\hline
\end{tabular}

Fig. 2. Classificação Hierárquica Descendente $(N=665)$.

\subsubsection{Classe 1 - Indústria pornográfica e aspetos negativos}

A primeira classe é a maior, com $45.64 \%$ do corpus, focando aspetos relativos a propagação, acesso e consumo de pornografia e seus potenciais aspetos negativos. Palavras desta classe foram enunciadas, sobretudo, por indivíduos do género feminino, com um posicionamento indiferente ou contra o consumo de pornografia e que consomem menos pornografia.

\subsubsection{Classe 2 - Categorias pornográficas}

A segunda classe é a menor, com $17.84 \%$ do corpus, referindo-se às secções presentes nos sites pornográficos, incluindo práticas sexuais e zonas corporais erógenas. Palavras desta classe foram referidas, sobretudo, por indivíduos do género masculino ou de outro género, 
com um posicionamento a favor do consumo de pornografia, e que consomem mais pornografia.

\subsubsection{Classe 3 - Propósitos do uso e aspetos positivos}

A última classe, com $36.51 \%$ do corpus, corresponde às finalidades do consumo de pornografia e potenciais aspetos positivos. Palavras desta classe foram indicadas, sobretudo, por indivíduos com um posicionamento a favor do consumo de pornografia, e que consomem menos pornografia.

Os resultados mostraram que as pessoas pensam em pornografia de diferentes formas. Pensam nos meios de consumo e potenciais consequências negativas (Classe 1), na diversidade de práticas e preferências sexuais presentes nos sites pornográficos (Classe 2), e nas finalidades do seu uso e potenciais aspetos positivos (Classe 3). A positividade das palavras foi moderada $(M=3.40, D P=0.94)$. As correlações revelaram que a positividade estava correlacionada positivamente com as atitudes favoráveis, $r=.62, p<.001$, e concordância com os efeitos positivos da pornografia, $r=.50, p<.001$; e, correlacionada negativamente com a concordância com os efeitos negativos da pornografia, $r=-.44, p<$ .001. Ao comparar a positividade em função do género encontrou-se diferenças significativas, $t(657)=-7.58, p<.001, d=0.61,95 \%$ IC [0.45,0.77], com o género masculino $(M=3.74, D P=0.90)$ a conotar mais positivamente as palavras mencionadas do que 0 género feminino $(M=3.19, D P=0.91)$.

\subsection{Consumo}

A análise descritiva do posicionamento face ao consumo de pornografia revelou que a maioria dos participantes é a favor ( $n=341$ : $51.28 \%$ ) ou indiferente ( $n=279: 41.95 \%$ ). Apenas uma diminuta percentagem se afirmou contra o consumo de pornografia $(n=45$ : $6.77 \%$ ). A análise descritiva do item sobre consumo de pornografia indicou que a maioria, quase $88 \%$ dos participantes já consumiu pelo menos uma vez pornografia $(n=581$ : $87.37 \%)$, e que apenas $12.63 \%$ dos participantes indicou nunca ter consumido $(n=84)$. De entre os que já consumiram pornografia $(n=581)$, a frequência de consumo é, na maioria, até duas/três vezes por mês $(n=335: 57.66 \%)$. 
Tabela 1. Média da Frequência de Consumo e das Atitudes Face à Pornografia em Função do Género

\begin{tabular}{|c|c|c|c|c|c|c|c|c|}
\hline \multirow[b]{2}{*}{ Variáveis } & \multicolumn{2}{|c|}{ Género $^{1}$} & \multirow[b]{2}{*}{$t$} & \multirow[b]{2}{*}{$g l$} & \multirow[b]{2}{*}{$p$} & \multirow[b]{2}{*}{$\begin{array}{c}d \text { de } \\
\text { Cohe } \\
n\end{array}$} & \multicolumn{2}{|c|}{$95 \%$ IC } \\
\hline & $\begin{array}{c}\text { Feminino } \\
M(D P)\end{array}$ & $\begin{array}{c}\text { Masculino } \\
M(D P)\end{array}$ & & & & & Inf. & Sup. \\
\hline Frequência de consumo ${ }^{2}$ & $\begin{array}{c}4.63 \\
(2.01)\end{array}$ & $7.07(1.86)$ & 15.02 & 552 & $\begin{array}{l}< \\
.001\end{array}$ & 1.26 & 1.08 & 1.44 \\
\hline $\begin{array}{l}\text { Atitudes face à } \\
\text { pornografia }^{3}\end{array}$ & $\begin{array}{l}3.27 \\
(0.90)\end{array}$ & $3.67(0.83)$ & 5.73 & 657 & .001 & 0.46 & 0.31 & 0.62 \\
\hline Efeitos positivos ${ }^{3}$ & $\begin{array}{l}3.26 \\
(0.86)\end{array}$ & $3.45(0.74)$ & 3.03 & 591 & .003 & 0.24 & 0.09 & 0.39 \\
\hline Efeitos negativos ${ }^{3}$ & $\begin{array}{l}2.90 \\
(0.74)\end{array}$ & $2.85(0.75)$ & 0.98 & 657 & .327 & - & - & - \\
\hline
\end{tabular}

Nota. Os valores em negrito indicam a maior média entre os grupos.

$M=$ média; $D P=$ desvio padrão

Quanto à relação entre o género e o consumo de pornografia, verificaram-se diferenças significativas. Com o género masculino a consumir pornografia com maior frequência do que o género feminino.

Quanto ao papel do género nas atitudes face à pornografia, também se encontraram diferenças significativas, com o género masculino a apresentar atitudes mais favoráveis e maior concordância com os efeitos positivos da pornografia do que o género feminino. Não emergiram diferenças significativas na concordância com os efeitos negativos da pornografia (Tabela 1).

\section{Discussão}

O recurso ao método misto, pelo entendimento multidimensional, abrangência e profundidade que oferece, permitiu melhor compreender como os significados, consumo e atitudes face à pornografia se relacionam com o género. A complementaridade dos dados qualitativos e quantitativos tornou a análise e conclusões derivadas mais completas e robustas. A análise qualitativa, através da técnica de associação livre de palavras, permitiu compreender os significados da pornografia ao captar as construções latentes da pornografia de forma profunda e pouco refletida. Percebeu-se o modo diferenciado como a pornografia é significada, o que corrobora que o significado da pornografia não é transversal (Brown et al., 2017; Hald et al., 2014). Ainda assim, sexo e nudez, bem como, masturbação e prazer foram aspetos centrais, o que vai ao encontro da definição de pornografia adotada.

\footnotetext{
${ }^{1}$ Dado o número de participantes desproporcional, analisamos apenas o género feminino $(n=403)$ e masculino $(n=206)$.

${ }^{2}$ Comparação realizada com os participantes que indicaram já ter consumido pornografia $(n=581)$.

${ }^{3}$ Comparação realizada com todos os participantes $(N=665)$.
} 
A análise mista permitiu analisar o papel do género na significação da pornografia, consumo e atitudes face à pornografia. Evidenciou-se que os significados diferem consoante o género. Assim como, o consumo e atitudes face à pornografia, o que vai ao encontro de investigações anteriores (Alferes, 1997; Brown et al., 2017; Cooper et al., 2012; Gomes, Fernandes, Ribeiro, \& Cardoso, 2018; Gomes, Fernandes, Ribeiro, Cardoso et al., 2018; Hald et al., 2014; Lam \& Chan, 2017; Lo \& Wei, 2005; Luquis et al., 2012; Raposo, 2018). Tais resultados podem espelhar considerações sociais ainda vigentes em Portugal, como o duplo padrão sexual e a veiculação de diferentes papéis de género, que impactam a expressão e vivência da sexualidade. Não obstante as diferenças de género, concluiu-se que o consumo de pornografia é uma realidade, o que vai ao encontro das estatísticas do Pornhub (Público, 2019).

\section{CONCLUSÃO}

O método misto permitiu obter conhecimento diferenciado sobre pornografia, revelando-se uma mais-valia na compreensão de fenómenos complexos. Concluiu-se que os significados, o consumo e atitudes face à pornografia estão relacionados com o género. Assim, importa integrar na abordagem da sexualidade e na educação sexual as questões de género, de modo a consciencializar, desconstruir estigmas e quebrar o duplo padrão sexual e mitos sexuais prejudiciais perpetuados, promovendo a vivência sexual saudável e igualitária entre os géneros. Apesar das limitações da amostra, jovem e muito escolarizada, este estudo contribuiu com investigação numa área onde esta escasseia e que importa continuar a aprofundar, pois o consumo de pornografia é uma realidade e tenderá a crescer. Futuramente, é relevante aprofundar as questões de género, por exemplo, através de grupos focais, na vivência do consumo de pornografia, desde significados a padrões de consumo e efeitos percebidos. E, continuar a investigar o modo como as considerações sociais prevalentes, como o duplo padrão sexual, impactam não só o consumo de pornografia como também a sexualidade globalmente. Recomenda-se, ainda, para estudos futuros, verificar se o género também estará relacionado com diferentes significados atribuídos a outros conceitos, como, por exemplo, "sexualidade", "sexo", "bom sexo" ou "satisfação sexual". 


\section{REFERÊNCIAS}

Abric, J. C. (2003). L'analyse structurale des représentations sociales. In S. Moscovici \& F. Buschini (Eds.), Les méthodes des sciences humaines (pp. 375-392). Paris: Presses Universitaires de France.

Alarcón, R., Iglesia, J., Casado, N., \& Montejo, A. (2019). Online porn addiction: What we know and what we don't - a systematic review. Journal of Clinical Medicine, 8(1), 91. doi:10.3390/jcm8010091

Alferes, V. R. (1997). Encenações e comportamentos sexuais: Para uma psicologia social da sexualidade. Porto: Edições Afrontamento.

Alves, M. P., Amâncio, L., \& Alferes, V. R. (2008). Género e representações sociais: Duplo-padrão sexual em função da religião e da posição política. Psicologia, 22(2), 139-160. doi:10.17575/rpsicol.v22i2.351

Blais-Lecours, S., Vaillancourt-Morel, M.-P., Sabourin, S., \& Godbout, N. (2016). Cyberpornography: Time use, perceived addiction, sexual functioning, and sexual satisfaction. Cyberpsychology, Behavior, and Social Networking, 19(11), 649-655. doi:10.1089/cyber.2016.0364

Brown, C. C., Durtschi, J. A., Carroll, J. S., \& Willoughby, B. J. (2017). Understanding and predicting classes of college students who use pornography. Computers in Human Behavior, 66, 114-121. doi:10.1016/j.chb.2016.09.008

Camargo, B. V., \& Justo, A. M. (2013). IRAMUTEQ: Um software gratuito para análise de dados textuais. Temas em Psicologia, 21(2), 513-518. doi:10.9788/TP2013.2-16

Carvalho, J., Czop, O., Rocha, M., Nobre, P., \& Soares, S. (2018). Gender differences in the automatic attention to romantic vs sexually explicit stimuli. Journal of Sexual Medicine, 15(8), 1083-1092. doi:10.1016/j.jsxm.2018.06.008

Carvalho, J., Gomes, A. Q., Laja, P., Oliveira, C., Vilarinho, S., Janssen, E., \& Nobre, P. (2013). Gender differences in sexual arousal and affective responses to erotica: The effects of type of film and fantasy instructions. Archives of Sexual Behavior, 42(6), 1011-1019. doi:10.1007/s10508-013-0076-2

Cooper, A. (1998). Sexuality and the Internet: Surfing into the new millennium. CyberPsychology \& Behavior, 1(2), 187-193. doi:10.1089/cpb.1998.1.187

Cooper, A., Morahan-Martin, J., Mathy, R. M., \& Maheu, M. (2002). Toward an increased understanding of user demographics in online sexual activities. Journal of Sex and Marital Therapy, 28(2), 105-129. doi:10.1080/00926230252851861

Esteves, M. (2018, abril). Tecnologia e sexo. At ShARE-UP, What can technology change? - Perspetivas, Faculdade de Engenharia da Universidade do Porto, Porto.

Gomes, A., Fernandes, A., Ribeiro, R., \& Cardoso, J. (2018). Ciberpornografia e atitudes sexuais em estudantes universitários: Estudo exploratório. In I. Leal, S. V. Humboldt, C. Ramos, A. Ferreira-Valente, \& J. L. P. Ribeiro (Eds.), $12^{\circ}$ Congresso Nacional de Psicologia da Saúde - Actas (pp. 33-42). Lisboa: Instituto Superior de Psicologia Aplicada.

Gomes, A., Fernandes, A., Ribeiro, R., Cardoso, J., \& Ramos, C. (2018). Perceived addiction to online pornography and sexual attitudes in Portuguese college students. Journal of Sexual Medicine, 15(3), 390390. doi:10.1016/j.jsxm.2018.04.586

Guerra, V. M., Andrade, F. C. B., \& Dias, M. R. (2004). Atitudes de estudantes universitários frente ao consumo de materiais pornográficos. Estudos de Psicologia, 9(2), 269-277. doi:10.1590/S1413-294X2004000200008

Guerra, V. M., Gouveia, V. V., Sousa, D. M., Lima, T. J., \& Freires, L. A. (2012). Sexual Liberalism-Conservatism: The effect of human values, gender, and previous sexual experience. Archives of Sexual Behavior, 41(4), 1027-1039. doi:10.1007/s10508-012-9936-4

Hald, G. M., Seaman, C., \& Linz, D. (2014). Sexuality and pornography. In D. Tolman, L. Diamond, J. Bauermeister, W. George, J. Pfaus, \& M. Ward (Eds.), APA Handbook of sexuality and Psychology: Contextual approaches (Vol. 2, pp. 3-35). Washington, DC: American Psychological Association.

Lam, C. B., \& Chan, D. K. S. (2007). The use of cyberpornography by young men in Hong Kong: Some 
psychosocial correlates. Archives of Sexual Behavior, 36(4), 588-598. doi:10.1007/s10508-006-9124-5

Lo, V., \& Wei, R. (2005). Exposure to Internet pornography and Taiwanese adolescents' sexual attitudes and behavior. Journal of Broadcasting \& Electronic Media, 49(2), 221-237. doi:10.1207/s15506878jobem4902_5

Lorvick, J., Bourgois, P., Wenger, L. D., Arreola, S. G., Lutnick, A., Wechsberg, W. M., \& Kral, A. H. (2012). Sexual pleasure and sexual risk among women who use methamphetamine: A mixed methods study. International Journal of Drug Policy, 23(5), 385-392. doi:10.1016/j.drugpo.2012.07.005

Luquis, R. R., Brelsford, G. M., \& Rojas-Guyler, L. (2012). Religiosity, spirituality, sexual attitudes, and sexual behaviors among college students. Journal of Religion and Health, 51(3), 601-614. doi:10.1007/s10943011-9527-z

Medium. (2017, 19 de fevereiro). How big is the porn industry? Disponível em https://medium.com/@Strange_bt_True/how-big-is-the-porn-industry-fbc1ac78091b

Morgan, E. M. (2011). Associations between young adults' use of sexually explicit materials and their sexual preferences, behaviors, and satisfaction. Journal of Sex Research, 48(6), 520-530. doi:10.1080/00224499.2010.543960

Pachankis, J. E., Buttenwieser, I. G., Bernstein, L. B., \& Bayles, D. O. (2013). A longitudinal, mixed methods study of sexual position identity, behavior, and fantasies among young sexual minority men. Archives of Sexual Behavior, 42(7), 1107-1368. doi:10.1007/s10508-013-0090-4

Pornhub. (2019, 11 de dezembro). The 2019 Year in Review. Disponível em https://www.pornhub.com/insights/2019-year-in-review

Público. (2019, 3 de abril). Pornhub: Portugal é um dos 40 países que mais vêem pornografia. Disponível em https://www.publico.pt/2019/04/03/p3/noticia/portugal-40-paises-ve-pornografia-1867936

Raposo, E. (2018). Relationship between pornography consumption and sexual health (dissertação de mestrado). Faculdade de Psicologia e de Ciências da Educação da Universidade do Porto, Porto.

Rasmussen, K. (2016). A historical and empirical review of pornography and romantic relationships: implications for family researchers. Journal of Family Theory and Review, 8, 173-191. doi:10.1111/jftr.12141

Ratinaud, P. (2009). IRAMUTEQ: Interface de $R$ pour les Analyses Multidimensionnelles de Textes et de Questionnaires [Computer software]. Disponível em http://www.iramuteq.org

Reiss, I. (1964). The scaling of premarital sexual permissiveness. Journal of Marriage and the Family, 26, 188198

Rosenberg, S., \& Jones, R. (1972). A method for investigating and representing a person's implicit theory of personality: Theodore Dreiser's view of people. Journal of Personality and Social Psychology, 22(3), 373386. doi:10.1037/h0032891

Short, M. B., Black, L., Smith, A. H., Wetterneck, C. T., \& Wells, D. E. (2012). A review of Internet pornography use research: Methodology and content from the past 10 years. Cyberpsychology, Behavior, and Social Networking, 15(1), 13-23. doi:10.1089/cyber.2010.0477

Small, M. L. (2011). How to conduct a mixed methods study: Recent trends in rapidly growing literature. Annual Review Sociology, 37, 57-86.

Tarrant, S. (2016). The pornography industry: What everyone needs to know. Oxford: Oxford University Press.

Træen, B., Nilsen, T. S., \& Stigum, H. (2006). Use of pornography in traditional media and on the Internet in Norway. The Journal of Sex Research, 43(3), 245-254. doi:10.1080/00224490609552323

Watson, M. A., \& Smith, R. D. (2012). Commentary - Positive porn: Educational, medical, and clinical uses. American Journal of Sexuality Education, 7(2), 122-145. doi:10.1080/15546128.2012.680861

Zaikman, Y., \& Marks, M. (2017). Promoting theory-based perspectives in sexual double standard research. Sex Roles, 76, 407-420. doi: 10.1007/s11199-016-0677-z 